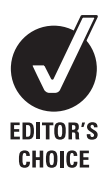

${ }^{1}$ Institute of Rheumatology, Department of Rheumatology of the First Faculty of Medicine, Charles University in Prague, Prague, Czech Republic;

${ }^{2}$ Rheumatology Department, Faculty Hospital Plzen, Plzen, Czech Republic; ${ }^{3}$ Institute of Biostatistics and Analyses, Masaryk Univerzity, Brno, Czech Republic

Correspondence to: Professor K Pavelka, Institute of Rheumatology, Na Slupi 4, Prague 2, 12850 Czech

Republic; pavelka@revma.cz

Accepted 3 March 2009 Published Online First 6 April 2009

\title{
Increasing the infliximab dose in rheumatoid arthritis patients: a randomised, double blind study failed to confirm its efficacy
}

\author{
K Pavelka, ${ }^{1} \mathrm{~K}$ Jarošová, ${ }^{1} \mathrm{D}$ Suchý, ${ }^{2} \mathrm{~L}$ Šenolt, ${ }^{1} \mathrm{~K}$ Chroust, ${ }^{3} \mathrm{~L}$ Dušek, ${ }^{3} \mathrm{~J}$ Vencovský ${ }^{1}$
}

\author{
ABSTRACT \\ Objective: To evaluate the effect of infliximab dose \\ escalation in incomplete responders in a randomised \\ controlled trial.
}

Methods: 141 rheumatoid arthritis (RA) patients treated with infliximab for 12 months (3 mg/kg; intervals 0, 2, 6 and then 8 weeks) who responded to the drug (disease activity score in 28 joints (DAS28) decrease $>1.2$ ) but who were not in remission (DAS28 >2.6) were enrolled into the study. Patients were randomly assigned into arm A, $3 \mathrm{mg} / \mathrm{kg}$, and arm B, $5 \mathrm{mg} / \mathrm{kg}$ infliximab every 8 weeks. Outcome measures included the DAS28, its components and C-reactive protein (CRP).

Results: There were no significant differences in changes in the DAS28, its components, or CRP in patients in arms $A$ and $B$ during the 12 months of treatment. All patients showed a DAS28 decrease greater than 0.6 after 28 weeks. Eleven patients interrupted therapy in arm A and 14 in arm B. Infusion reactions and non-serious adverse events were observed in $4.2 \%$ and $28.2 \%$ of arm A patients and in $7.2 \%$ and $47.8 \%$ of arm B patients. The frequency of serious adverse events was comparable between arms A and B (16.9\% and $15.9 \%$, respectively), and the frequency of serious infections was not significantly greater in the higher dose group (5.8\%) than in the lower dose group (5.6\%).

Conclusions: In this setting, increasing the infliximab dose from $3 \mathrm{mg} / \mathrm{kg}$ to $5 \mathrm{mg} / \mathrm{kg}$ in RA patients with residual disease activity did not improve efficacy but moderately increased toxicity. These data indicate that a switch to another biological treatment would be a more appropriate strategy in incomplete responders.

Infliximab is a chimeric monoclonal antibody against tumour necrosis factor (TNF) alpha. In 1999, it was approved for use in combination with methotrexate for treating rheumatoid arthritis (RA). Clinical studies demonstrated clinical efficacy, an effect on radiological progression and the relative safety of long-term infliximab administration in RA patients. ${ }^{1-3}$ The Anti-TNF Trial in Rheumatoid Arthritis with Concomitant Therapy (ATTRACT) study demonstrated the efficacy of a $3 \mathrm{mg} / \mathrm{kg}$ dose administered at 0, 2 and 6 weeks, followed by administration every 8 weeks. ${ }^{4}$ Consequently, the US Food and Drug Administration and the European Medicines Agency approved this dose of infliximab for use in RA patients.

Clinical practice has shown that not all patients respond to the $3 \mathrm{mg} / \mathrm{kg}$ dose of infliximab, leading some doctors to use higher doses. In a retrospective analysis of two cohort studies, Stern and Wolfe ${ }^{5}$ demonstrated that infliximab doses were consistently increased in $61 \%$ and $56 \%$ of patients, respectively, with a median time of 7 months before the dose was increased. The most common reasons for the dose increase were insufficient response, too short an effect when administered every 8 weeks, or a decreased response to the drug following the initial response. Ariza-Ariza et $a l^{6}$ reported similar findings in an analysis of 16 studies involving 8510 patients. In those studies, the doses were increased in $53.7 \%$ of patients. The question of whether an increased dose of infliximab is effective in partial responders has been analysed: several observational studies ${ }^{7-9}$ found a predominantly positive effect, as did the START study, which was a prospective double blind study. ${ }^{10}$ Based on these studies, in March 2007 the European Medicines Agency amended the text of the infliximab information brochure, which now indicates that infliximab may be used for RA treatment in combination with methotrexate. The brochure specifies administering infliximab every 8 weeks at an initial dose of $3 \mathrm{mg} / \mathrm{kg}$; the dose is then titrated up to $7.5 \mathrm{mg} / \mathrm{kg}$ and administered every 8 weeks. Alternatively, a dose of $3 \mathrm{mg} /$ $\mathrm{kg}$ may be administered at shorter 4-week intervals.

There is little evidence of the effectiveness of the dose increase in terms of a prospective randomised study. Therefore, our aim was to perform a controlled, randomised double blind study to determine whether an increase in infliximab dose from $3 \mathrm{mg} / \mathrm{kg}$ to $5 \mathrm{mg} / \mathrm{kg}$ results in a clinically positive effect.

\section{METHODS}

\section{Setting and participants}

In the Czech Republic, all patients receiving biological treatments are entered into the ATTRA Registry. ${ }^{11}$ Biological treatment is indicated based on the recommendations of the Czech Society of Rheumatology. ${ }^{12}$ Indication criteria include the following: failure of at least two disease-modifying antirheumatic drugs; disease activity, expressed as a disease activity score in 28 joints (DAS28) greater than 5.1; and no contraindications for anti-TNF $\alpha$ use, including negative results when screened for latent tuberculosis. Criteria for inclusion into this clinical trial included listing in the ATTRA registry for 12 months, ie, 12 months of treatment with a combination of methotrexate and $3 \mathrm{mg} / \mathrm{kg}$ infliximab administered at weeks 0, 2, 6, 8 and every 8 weeks thereafter. Such patients must have had a positive response to infliximab treatment within 
the first 3 months, defined as a DAS28 decrease of greater than 1.2 compared with the baseline level. To be included, patients also had to be partial responders or experience reduced effectiveness over the course of treatment; in other words, at the time of enrolment in our study, and after 12 months of treatment as noted above, the patients had to have a DAS28 greater than 2.6.

Screening for latent tuberculosis was performed before infliximab was introduced to patients, ie, when the patients entered the ATTRA Registry. The criteria required a negative tuberculosis history, negative chest $x$ ray and a tuberculin test result of less than $10 \mathrm{~mm}$. Patients not fulfilling these criteria were excluded from treatment, and no isoniazid was used before infliximab. The screening was not repeated before enrolment in the dose escalation study. The study protocol was approved by a multicentre ethics committee, and all patients provided informed written consent for inclusion in the dose escalation study.

Subjects were randomly assigned to trial arms A and B using a simple block randomisation procedure with three stratification criteria: duration of RA disease ( $\leqslant 2$ years, $>2$ years); baseline DAS28 $(<4.5,4.5-5.5,>5.5)$ and swollen joint count $(<6, \geqslant 6)$. Patients were enrolled in medical centres that administered biological therapy. Randomisation took place at the Institute of Biostatistics and Analyses. The first patient was randomly assigned on 14 August 2003, and the last patient was randomly assigned on 22 April 2005. Eleven patients were randomly assigned who had had RA for 2 years or less. In the group who had had the disease for more than 2 years, there were 65 patients with a baseline DAS28 less than 4.5, 48 with a baseline DAS28 4.5-5.5 and 17 patients with a DAS28 greater than 5.5.

Infliximab was administered intravenously at a dose of $3 \mathrm{mg} /$ $\mathrm{kg}$ every 8 weeks in arm A and $5 \mathrm{mg} / \mathrm{kg}$ every 8 weeks in arm B by a study nurse, who was aware of the dosing. Both the investigator and patient were blinded to the study treatment (dose).

\section{Concomitant treatment}

All patients were treated with methotrexate, which was administered at a stable dose throughout the study. The doses of oral glucocorticoids were also stable. Intra-articular administration of glucocorticoids and on-demand non-steroidal antiinflammatory drugs were allowed as required but were not encouraged.

\section{Study endpoints}

The primary study endpoint was a change in the DAS2 $8^{13}$ after 28 weeks compared with the trial entry value. Secondary endpoints were a change in the number of swollen and tender joints and changes in the DAS28, C-reactive protein (CRP) and erythrocyte sedimentation rate. These values were evaluated at weeks 8, 16, 24, 28, 32, 40, 48 and 52 .

\section{Statistical analysis}

Standard descriptive statistics were used to express differences among subgroups of cases (relative frequencies for categorical variables and arithmetic means and standard deviations). Differences between arms were determined by Fisher's exact test for binary outcomes. The ML chi-square test was used to test for ordinal categorical variables, and the two-sample t test with equal variances or the Mann-Whitney test was used for continuous variables. Analysis of variance was used for evaluating time-related changes in the study endpoints and repeated measures. Analysis of covariance (ANCOVA) was used to examine statistical differences between groups adjusted for the baseline values. The $t$ test was used to determine the statistical significance of individual parameters in the model. The $\mathrm{F}$ test was used to compare two different ANCOVA models. A two-sided $p$ value less than 0.05 was considered statistically significant.

A sample size calculation was performed with an alpha of 0.05 , an expected standard deviation of $0.8-1.0$ and a power of $90 \%$. We considered a difference of 0.6 in the DAS28 to be clinically relevant. Using the Mann-Whitney test to compare the DAS28 difference 28 weeks after randomisation between arms, we found that a total of 70 patients were needed for each arm. This allowed for a decrease of approximately 10\% in the number of patients. Intent-to-treat analyses with the last observation carried forward (LOCF) were used to compare the two treatment arms.

The data were analysed using the Statistica data analysis software system, version 8.0 and R (R Development Core Team, 2006).

\section{RESULTS}

A total of 141 patients was randomly assigned into the study: 71 patients into arm A (3 mg/kg infliximab) and 70 patients into $\operatorname{arm~B~}(5 \mathrm{mg} / \mathrm{kg}$ infliximab). In $\operatorname{arm~B}$, one patient was randomly assigned twice, so that 71 patients in arm $A$ and 69 in arm B were evaluated (fig 1). In arm A, 60 patients completed the study (12 months). Of the 11 premature withdrawals, four were due to adverse events, two were due to insufficient efficacy and two were the patient's decision; other reasons were planned gravidity and proteinuria. In arm B, a total of 14 patients failed to finish the study: five withdrawals were due to adverse events, two to insufficient efficacy and three were the patient's decision; other reasons were planned gravidity and withdrawal of consent.

There were no differences between arms A and B with regard to any demographic or activity indicators at the beginning of the study (table 1). Baseline activity was consistent with that of other patients who are partial responders to infliximab.

\section{Study endpoints}

During the first year of treatment, ie, during the first 12 months of entry into the ATTRA Registry, the DAS28 decreased in patients who were later enrolled into arm A from 6.7 (95\% CI

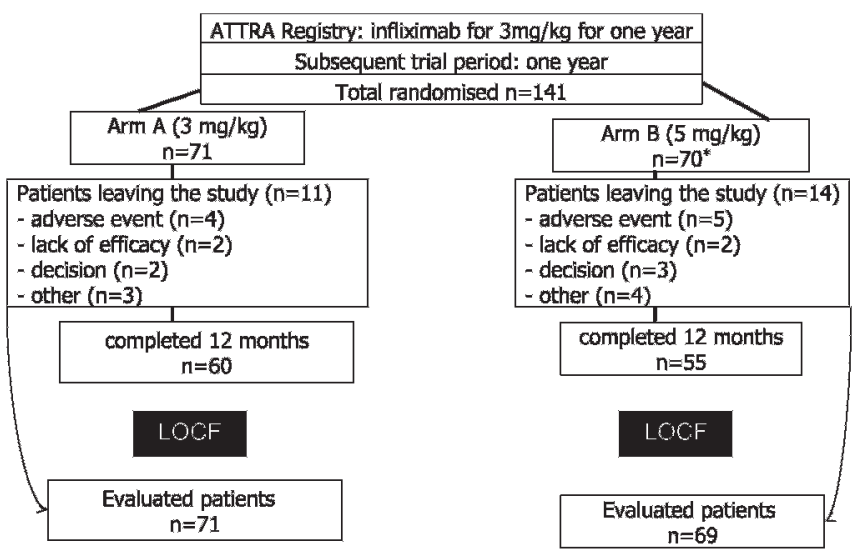

Figure 1 Overview of the randomised trial of the increased infliximab dose. LOCF, last observation carried forward. 
Table 1 Baseline demographic and clinical characteristics of RA patients treated with infliximab

\begin{tabular}{lll}
\hline & $\begin{array}{l}\text { Arm A } \\
(\mathbf{3} \mathbf{~ m g} / \mathbf{k g}) \\
(\mathbf{n}=\mathbf{7 1})\end{array}$ & $\begin{array}{l}\text { Arm B } \\
\mathbf{( 5 ~} \mathbf{~ m g} / \mathbf{k g}) \\
(\mathbf{n}=\mathbf{6 9})\end{array}$ \\
\hline Age, mean years (SD) & $45.6(13.2)$ & $48.1(13.3)$ \\
Gender, female/male, \% & $67 / 33$ & $69 / 31$ \\
Disease duration, mean years (SD) & $13.0(8.1)$ & $12.9(8.4)$ \\
Glucocorticoids & $52 \%$ & $51 \%$ \\
Baseline DAS28, at study randomisation; mean & $4.5(1.1)$ & $4.5(1.2)$ \\
DAS28 (SD) & $4.8(3.6)$ & $4.5(3.6)$ \\
Swollen joints, mean count (SD) & $14.2(4.9)$ & $14.1(4.7)$ \\
Methotrexate dose, mean dose (SD) & $3.4(0.5)$ & $5.1(0.5)$ \\
Infliximab dose in the study, mg/kg; mean dose (SD)
\end{tabular}

DAS28, disease activity score in 28 joints; RA, rheumatoid arthritis; SD, standard deviation.

6.6 to 6.9 ) to 4.3 (95\% CI 4.1 to 4.6 ) and in patients enrolled into arm B from 6.5 (95\% CI 6.3 to 6.7 ) to 4.1 (95\% CI 3.9 to 4.3 ). Patients were then randomly assigned into arm A $(3 \mathrm{mg} / \mathrm{kg}$ infliximab) and $\operatorname{arm~B~(5~mg/kg~infliximab).~In~arm~} \mathrm{A}$, the DAS28 was 4.5 (95\% CI 4.2 to 4.8$)$ at the beginning of the study and 4.0 (95\% CI 3.7 to 4.3$)$ at the end of the treatment at week 52 ; in arm B, the initial DAS28 values were 4.5 (95\% CI 4.2 to 4.8) and the final DAS28 values after week 52 were 3.9 (95\% CI 3.6 to $4.3 ; p=0.748)$. The change in DAS28 after 28 weeks compared with the trial entry value was thus 0.67 (95\% CI 0.42 to 0.92$)$ in $\operatorname{arm~A}$ and 0.65 in $\operatorname{arm~B~(95\% ~CI~} 0.40$ to 0.89 ; $\mathrm{p}=0.913)$.

No significant differences between the two groups were found at any other time point in the study (fig 2). In addition, statistical analysis of arms $A$ and $B$ did not reveal any differences in secondary endpoints (swollen joints, tender joints, erythrocyte sedimentation rate, CRP; fig 3).

The DAS28 and CRP were examined using ANCOVA. First, using the baseline DAS28 at the beginning of the study as the covariate and the trial arm as a factor, interactions between the baseline DAS28 and the arm were evaluated. In this model, the interactions did not show a significant effect $(p=0.811)$ and the

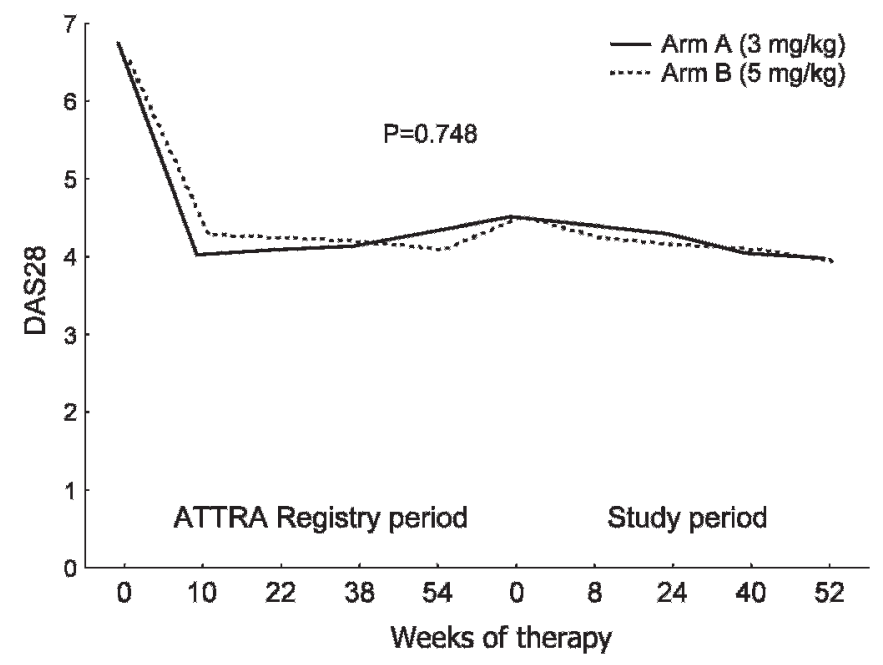

Figure 2 Mean change in the disease activity score in 28 joints (DAS28) in trial arms A and B over the course of the study. Patients in arm $A$ received $3 \mathrm{mg} / \mathrm{kg}$ infliximab and patients in arm $B$ received $5 \mathrm{mg} /$ $\mathrm{kg}$ infliximab. Last observation carried forward was used.

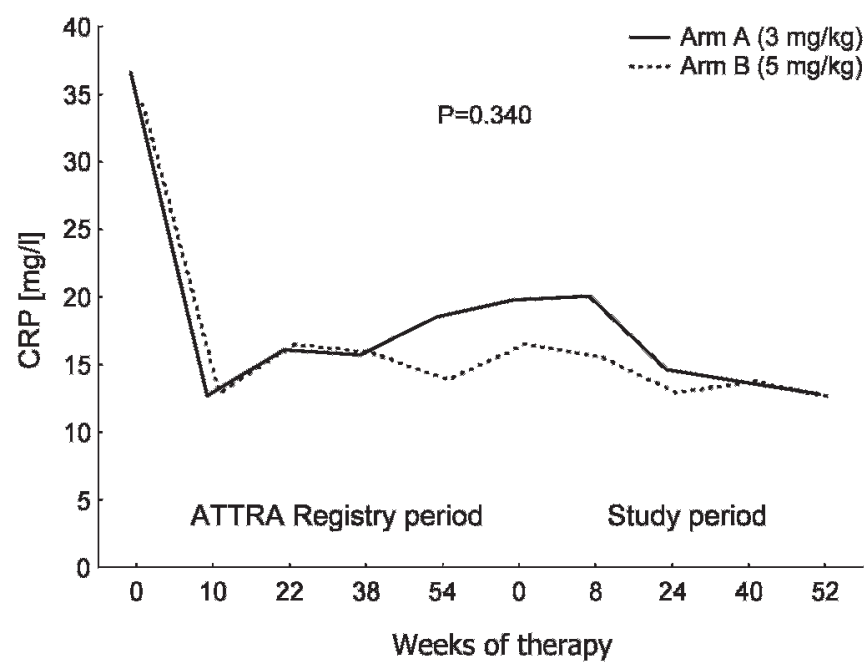

Figure 3 Mean change in C-reactive protein (CRP) over time in trial arms $A$ and $B$ over the course of the study. Patients in arm $A$ received $3 \mathrm{mg} / \mathrm{kg}$ infliximab and patients in arm B received $5 \mathrm{mg} / \mathrm{kg}$ infliximab. Last observation carried forward was used.

model without interactions was not significantly different from the model with interactions $(p=0.811)$, so a model without interactions was used. Using this model, the baseline DAS28 significantly correlated with the DAS28 at the end of the study $(p<0.001)$. This means that patients with a higher baseline DAS28 also had a higher DAS28 at week 52. There were no differences between arms $A$ and $B(p=0.831$; fig 4). First, using baseline CRP at the beginning of the study as the covariate and the trial arm as a factor, interactions between baseline CRP and the arm were evaluated. In this model, the interactions did not show a significant effect $(p=0.858)$ and the model without interactions was not significantly different from the model with interactions $(p=0.858)$, so a model without interactions was used. Using this model, the baseline CRP significantly correlated with the CRP at the end of the study $(p<0.001)$. This means that patients with higher baseline CRP values also had higher CRP values at week 52 . There were no differences between arms $A$ and $B(p=0.821$; fig 5).

\section{Side effects}

Infliximab was generally well tolerated throughout the study. Four patients in arm A (receiving $3 \mathrm{mg} / \mathrm{kg}$ infliximab) and five patients in arm B (receiving $5 \mathrm{mg} / \mathrm{kg}$ infliximab) discontinued the treatment due to adverse events. Non-serious adverse events were observed in $28.2 \%$ of arm A patients and in $47.8 \%$ of $\operatorname{arm~B}$ patients $(p=0.023)$. In $\operatorname{arm} A$, there were 15 serious adverse events in 12 patients $(16.9 \%)$; in arm B, there were 13 serious adverse events in 11 patients (15.9\%). Six infusion reactions were observed in three patients (4.2\%) in arm A and six were observed in five patients $(7.2 \%)$ in arm B $(p=0.490)$. Serious infections were reported in four patients in arm A (5.6\%) and in four patients $(5.8 \%)$ in arm B (there were two serious infections in one of the patients). There was no statistically significant difference in the incidence of serious infections between arms $\mathrm{A}$ and $B(p=1.000)$. One case of tuberculosis was detected in each group. In both cases, the diagnosis was disseminated tuberculosis; both patients died. Both patients were screened for latent tuberculosis before receiving their first dose of infliximab through the ATTRA Registry and had negative results. 


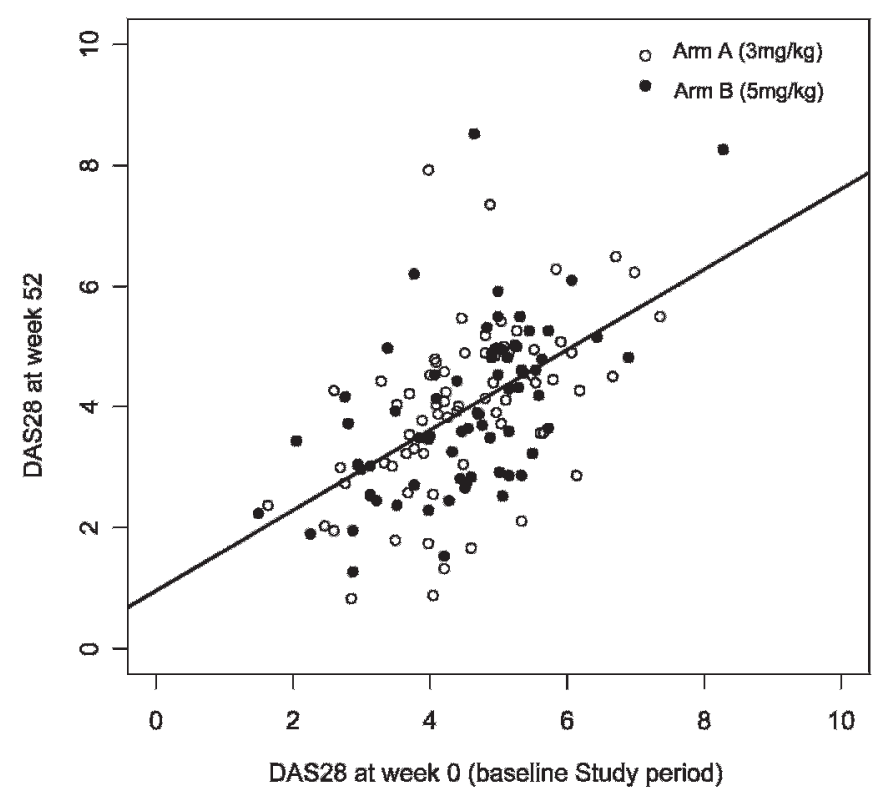

Figure 4 Disease activity score in 28 joints (DAS28) at week 0 and week 52 in trial arms $A$ and $B$. Patients in arm A received $3 \mathrm{mg} / \mathrm{kg}$ infliximab and patients in arm $B$ received $5 \mathrm{mg} / \mathrm{kg}$ infliximab.

\section{DISCUSSION}

In the setting of this randomised double blind controlled study, we failed to confirm that an increase in infliximab from 3 to $5 \mathrm{mg} / \mathrm{kg}$ had a long-term benefit for patients with RA. Patients who showed only partial response to standard infliximab treatment during the first year were randomly assigned into two arms for a second year of treatment; arm A continued at the same dose ( $3 \mathrm{mg} / \mathrm{kg}$ every 8 weeks), whereas in arm B, the dose was increased to $5 \mathrm{mg} / \mathrm{kg}$ every 8 weeks. The DAS28 showed only mild improvement, with no statistically significant difference between the two arms. Similarly, individual components of the DAS28 or CRP were not different between the two arms.

Van Vollenhoven et al demonstrated that an increase in the infliximab dose in partial responders was effective and led to a decrease in the DAS28. However, when they compared the best DAS28 during treatment with lower and higher doses, no difference was detected. Van der Cruyssen et al ${ }^{14}$ provided evidence that a positive effect could be achieved using a temporary dose increase that could subsequently be lowered to the initial dosage. A subgroup of patients, who had a decreased response to $3 \mathrm{mg} / \mathrm{kg}$ per 8 weeks of infliximab during the first 22 weeks of treatment, could increase their response to initial levels by adding $100 \mathrm{mg}$ infliximab to subsequent doses. ${ }^{7}$ Four-year survival rates for patients on infliximab were identical for patients who received and those who did not receive a dose increase. ${ }^{14}$ As most patients were back on the $3 \mathrm{mg} / \mathrm{kg}$ every 8 weeks dose regimen at that time point, this indicated that temporary infliximab dose escalation could correct a suboptimal response of patients to the $3 \mathrm{mg} / \mathrm{kg}$ dosage. A study by Sidiropoulos et $a^{15}$ provided interesting data about the effectiveness of infliximab dose escalation in regular clinical practice. Decisions made with respect to the dose increase in the study were made based strictly on an evaluation of RA activity using the disease activity score in 44 joints (DAS44). In patients with residual activity, as demonstrated by a DAS44 greater than 2.4, the methotrexate and/or infliximab dose was adjusted. The methotrexate/infliximab dose was increased in almost $80 \%$ of

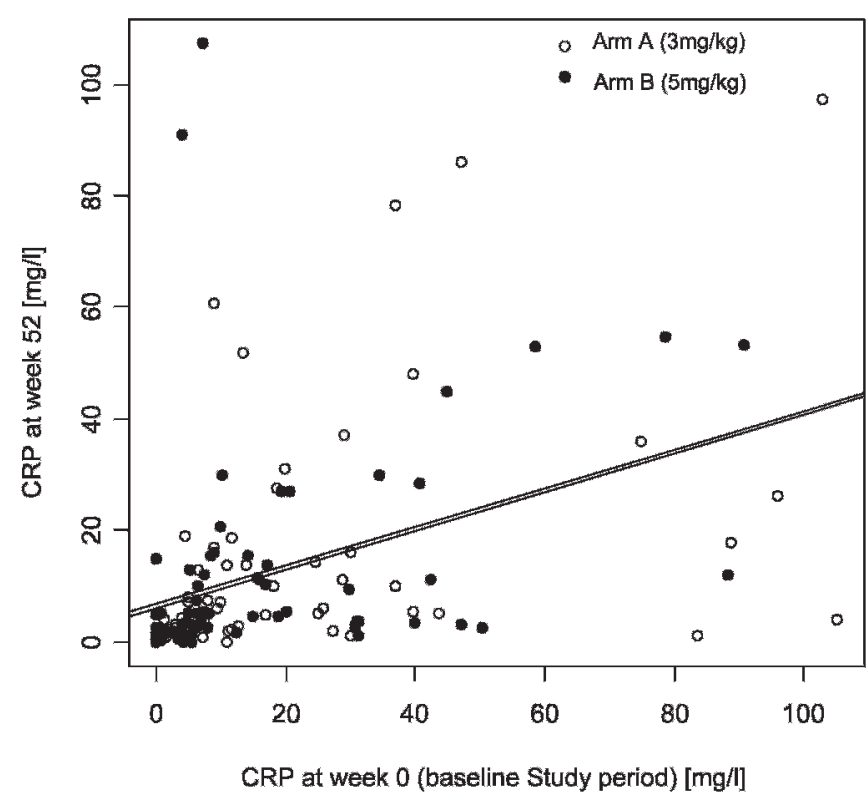

Figure 5 C-reactive protein (CRP) at week 0 and week 52 in trial arms $A$ and $B$. Patients in arm $A$ received $3 \mathrm{mg} / \mathrm{kg}$ infliximab and patients in arm $B$ received $5 \mathrm{mg} / \mathrm{kg}$ infliximab.

patients, resulting in a significant DAS44 decrease. However, the EULAR response improved in only $26 \%$ of patients.

Rahman et $a l^{11}$ studied the effect of an infliximab dose increase on clinical response, infliximab serum levels and the presence of anti-infliximab antibodies. Only $30 \%$ of the studied patients fulfilled the criteria for infliximab dose increase; the majority of patients responded to the initial dosage. On average, patients who required a dose increase had lower serum infliximab levels before the increase compared with patients in whom no dose adjustment was necessary. The dose increase resulted in an increase of serum infliximab levels, and $80 \%$ of the patients experienced a $20 \%$ improvement in the number of swollen and tender joints. Anti-infliximab antibodies did not seem to be the main reason for the decreased effectiveness of infliximab, and the majority of patients with anti-infliximab antibodies had a positive response to the dose increase.

It should be noted that there are other alternatives to infliximab when treatment is ineffective. For example, patients may be switched to another anti-TNF $\alpha$ agent, ${ }^{16}{ }^{17}$ to rituximab ${ }^{18}$ or to abatacept. ${ }^{19}$

We observed one case of tuberculosis in each study group (arms A and B). Patients treated for the first time with TNF blocking agents from 2001 to 2004 who were included in the Registry were screened according to guidelines of the Czech Rheumatological Society, which was used during this time period. This screening consisted of a medical history, chest $x$ ray and tuberculin skin test less than $10 \mathrm{~mm}$. Currently, the Czech Rheumatological Society is suggesting new guidelines for screening for latent tuberculosis, which would include the QuantiFERON-TB test. After the dose escalation, the incidence of serious infections and infusion reactions was unchanged; however, the rate of non-serious adverse events was significantly increased. These data are in agreement with the metaanalysis by Bongartz et al. ${ }^{20}$

The present study has some limitations. First, the design of the study and particularly the dosage regimen was rigid as a result of the fact that it was not a company-commissioned study; rather, it was a study performed on ATTRA Registry 
patients. Health insurance companies require that a strict dosage regimen be used, and this did not allow us to use more flexible dosing during the first year of the treatment. A second limitation of the study was that the maximum dose was $5 \mathrm{mg} /$ $\mathrm{kg}$ every 8 weeks. In fact, some clinicians use a dose of $7.5 \mathrm{mg} /$ $\mathrm{kg}$. However, at this point, it is also necessary to consider the economic impact of the treatment and the possible increased risk of infections.

The dosages at week 0 were in the range of $2.7-5.5 \mathrm{mg} / \mathrm{kg}$ in arm A patients and 3.3-6.3 mg/kg in arm B patients. In arm A, 18 patients did not comply with the prescribed dosage; in arm $\mathrm{B}$, four patients did not comply; this was approximately a $25 \%$ difference in non-compliance. For this reason, an analysis of patients who did comply with the prescribed dosage was performed that allowed for the $25 \%$ difference. The results were not different from those obtained from the analysis of the complete intent-to-treat population. No differences between arms were found. There were also no statistical differences between arms when we analysed the study completers.

In conclusion, our randomised controlled study did not confirm the effectiveness of an increased dose of infliximab from 3 to $5 \mathrm{mg} / \mathrm{kg}$ administered every 8 weeks in patients undergoing long-term treatment with infliximab. A decrease in the DAS28 greater than 0.6 was achieved in both arms of the trial, but there were no statistical differences between arms. This conclusion is valid for the current setting, but may not be valid in other settings or subpopulations of RA patients.

Funding: The study was supported by a grant of the Ministry of Health of the Czech Republic no VZ 00023728.

Competing interests: None.

Ethics approval: The study protocol was approved by a multicentre ethics committee.

Patient consent: Obtained.

\section{REFERENCES}

1. Maini RN, Breedveld FC, Kalden JR, Smolen JS, Davis D, Macfarlane JD, et al. Therapeutic efficacy of multiple intravenous infusions of anti-tumor necrosis factor alpha monoclonal antibody combined with low-dose weekly methotrexate in rheumatoid arthritis. Arthritis Rheum 1998;41:1552-63.

2. Lipsky PE, van der Heijde DM, St Clair EW, Furst DE, Breedveld FC, Kalden JR, et al, Anti-Tumor Necrosis Factor Trial in Rheumatoid Arthritis with Concomitant Therapy Study Group. Infliximab and methotrexate in the treatment of rheumatoid arthritis. N Engl J Med 2000;343:1594-602.

3. St Clair EW. Infliximab treatment for rheumatic disease: clinical and radiological efficacy. Ann Rheum Dis 2002;61(Suppl 2):ii67-9.

4. Maini R, St Clair EW, Breedveld F, Furst D, Kalden J, Weisman M, et al. Infliximab (chimeric anti-tumour necrosis factor alpha monoclonal antibody) versus placebo in rheumatoid arthritis patients receiving concomitant methotrexate: a randomised phase III trial. ATTRACT Study Group. Lancet 1999;354:1932-9.

5. Stern R, Wolfe F. Infliximab dose and clinical status: results of 2 studies in 1642 patients with rheumatoid arthritis. J Rheumatol 2004;31:1538-45.

6. Ariza-Ariza R, Navarro-Sarabia F, Hernández-Cruz B, Rodríguez-Arboleya L, NavarroCompán V, Toyos J. Dose escalation of the anti-TNF-alpha agents in patients with rheumatoid arthritis. A systematic review. Rheumatology (Oxford) 2007;46:529-32.

7. Durez P, Van den Bosch F, Corluy L, Veys EM, De Clerck L, Peretz A, et al. A dose adjustment in patients with rheumatoid arthritis not optimally responding to a standard dose of infliximab of $3 \mathrm{mg} / \mathrm{kg}$ every 8 weeks can be effective: a Belgian prospective study. Rheumatology (Oxford) 2005;44:465-8.

8. Van Vollenhoven RF, Brannemark S, Klareskog L. Dose escalation of infliximab in clinical practice: improvements seen may be explained by a regression-like effect. Ann Rheum Dis 2004;63:426-30.

9. Flendrie $\mathbf{M}$, Creemers MC, van Riel PL. Titration of infliximab treatment in rheumatoid arthritis patients based on response patterns. Rheumatology (Oxford) 2007:46:146-9.

10. Pavelka K, Gatterová J, Tegzová D, Jarosová K, Tomasová Studýnková J, Svobodník A, et al. Radiographic progression of rheumatoid arthritis in patients from the Czech National Registry receiving infliximab treatment. Clin Exp Rheumatol 2007;25:540-5

11. Rahman MU, Strusberg I, Geusens P, Berman A, Yocum D, Baker D, et al. Doubleblinded infliximab dose escalation in patients with rheumatoid arthritis. Ann Rheum Dis 2007;66:1233-8.

12. Vencovský J, Tegzová D, Krofta K, Pavelka K. Czech Society for Rheumatology guidelines for biological treatment with TNF blocking agents - supplement of standard treatment protocol in rheumatoid arthritis (in Czech). Ces Revmatol 2004;12:20-9.

13. Prevoo ML, van Gestel $A M$, van $T$ Hof $M A$, van Rijswijk $M H$, van de Putte $L B$, van Riel PL. Remission in a prospective study of patients with rheumatoid arthritis. American Rheumatism Association preliminary remission criteria in relation to the disease activity score. Br J Rheumatol 1996;35:1101-5.

14. Van der Cruyssen B, Westhovens R, Durez P, Van den Bosch F, Mielants H, Declerck $L$, et al. Tapering infliximab dosages after temporary dose increase does not effect treatment survival in an established RA population. Ann Rheum Dis 2007;66(Suppl II):193-4

15. Sidiropoulos P, Bertsias G, Kritikos HD, Kouroumali H, Voudouris K, Boumpas DT. Infliximab treatment for rheumatoid arthritis, with dose titration based on the Disease Activity Score: dose adjustments are common but not always sufficient to assure sustained benefit. Ann Rheum Dis 2004;63:144-8.

16. Burmester GR, Mariette X, Montecucco C, Monteagudo-Sáez I, Malaise M, Tzioufas $A G$, et al. Adalimumab alone and in combination with disease-modifying antirheumatic drugs for the treatment of rheumatoid arthritis in clinical practice: the Research in Active Rheumatoid Arthritis (ReAct) trial. Ann Rheum Dis 2007;66:732-9

17. Gomez-Reino JJ, Carmona L, BIOBADASER Group. Switching TNF antagonists in patients with chronic arthritis: an observational study of 488 patients over a four-year period. Arthritis Res Ther 2006;8:R29.

18. Finckh A, Ciurea A, Brulhart L, Kyburz D, Möller B, Dehler S, et al, Physicians of the Swiss Clinical Quality Management Program for Rheumatoid Arthritis. B cell depletion may be more effective than switching to an alternative anti-tumor necrosis factor agent in rheumatoid arthritis patients with inadequate response to anti-tumor necrosis factor agents. Arthritis Rheum 2007;56:1417-23.

19. Genovese MC, Becker JC, Schiff M, Luggen M, Sherrer Y, Kremer J, et al. Abatacept for rheumatoid arthritis refractory to tumor necrosis factor alpha inhibition. N Engl J Med 2005;353:1114-23.

20. Bongartz T, Sutton AJ, Sweeting MJ, Buchan I, Matteson EL, Montori V. Anti-TNF antibody therapy in rheumatoid arthritis and the risk of serious infections and malignancies: systematic review and meta-analysis of rare harmful effects in randomized controlled trials. JAMA 2006;295:2275-85. 